Ann. Zootech., I964, 13 (2), I99-2I2.

\title{
DESCRIPTION DE DEUX APPAREILS PERMETTANT LE CONTROLE AUTOMATIQUE DES DÉBITS DE LAIT AU COURS DE LA TRAITE A LA MACHINE PREMIERS RÉSULTATS OBTENUS CHEZ LA BREBIS
}

\author{
J. LABUSSIÈRE et J. MARTINET \\ avec la collaboration technique de P. I'etrequin, P. Lascaray. et B. Mirman \\ Laboratoire de Physiologie de la Lactation, \\ Centre national de Recherches zootechniques, Jouy-en-Josas (Seine-et-Oise)
}

\section{SOMMAIRE}

L'enregistrement automatique des courbes de descente de lait a été effectué lors de la traite à la machine de 23 brebis aux environs du premier mois de la lactation.

Deux techniques furent utilisées :

- l'une volumétrique, à l'aide d'un suiveur de niveau;

- l'autre pondérale, à l'aide d'un dynamomètre électronique.

- Trois brebis ont donné leur lait en une seule émission.

- Vingt brebis, par contre, présentent deux émissions bien distinctes.

Dans ce cas : la première émission correspondrait à l'écoulement du lait citernal. Elle présente son débit maximum vers la huitième seconde, après la pose des gobelets, puis diminue graduellement pour cesser vers la $24^{\mathrm{e}}$ seconde.

La seconde émission serait la phase d'écoulement du lait alvéolaire chassé des acinis par le réflexe neuro-endocrinien d'éjection. Elle commence vers la $30^{\mathrm{e}}$ seconde et se prolonge un peu au-delà de la première minute de traite.

Il existe de grandes différences individuelles dans la répartition du lait entre les différentes fractions, mais d'un jour à l'autre l'allure des courbes est stable chez un même animal.

L'élasticité de l'orifice du trayon jouerait un rôle limitant sur le débit.

\section{I. - INTRODUCTION}

La connaissance de la cinétique d'émission du lait au cours du processus de traite permet de juger, entre autres, de l'aptitude de l'animal à donner son lait et de la qualité du stimulus appliqué par le trayeur ou par la machine. De telles études ont surtout été effectuées chez la vache à l'aide de techniques pondérales et volumétriques. 


\section{Méthodes pondérales.}

I a suspension du pot trayeur à un dynamomètre est une méthode très employée, le contrôle des quantités de lait s'accumulant dans le récipient se faisant, selon les auteurs, toutes les io à 30 secondes (MaTTHEWs, Shaw et WEaVER, I928; FoOT, I935; Dodd et al., I950; GrígoIre et al., I954; BEIGEL, I955, CARUolo et al., I935; Holthoff, I956 ; Stewart et Schultz, I956; Brau, I956; Hupp et Plum, I957 ; Sandvik, I957 ; Schwenzer, I958 ; Joha.ssson et Malven, I960, etc.). Cette technique exige de l'opérateur une lecture rapide de la mesure et son enregistrement dans le court espace de temps qui lui est imparti. Elle est sujette à des erreurs importantes.

Afin de faciliter les opérations et d'améliorer l'exactitude des résultats, de nombreux auteurs, dont le premier fut GAINEs (I927), ont imaginé divers appareils à enregistrement automatique. Parmi ceux-ci on peut citer différents ensembles de transmission mécanique permettant d'inscrire sur un cylindre enregistreur l'extension du ressort d'un dynamomètre (BECK, FrYer et ROARK, I95I ; HUPFAUER, I956 ; ACE et al., I959; IONALD, I960; SyCh, I96I ; Viljoen et SWART, I96r).

WhitTliestone et Phirirtps (I953), Murray (I960) ont proposé des enregistreurs électriques plus perfectionnés. CLOUGH et DODD, I957, emploient un dispositif du type jauge de contrainte; enfin, WHITTIESTONE (I960) décrit un système de mesure par variation potentiométrique.

\section{Méthodes volumétriques.}

Flles consistent à noter à des intervalles de temps égaux les volumes de lait s'élevant dans un pot collecteur gradué ; dans le cas où le pot est muni de 4 compartiments, il est possible de suivre individuellement la cinétique de descente du lait de chaque quartier. Ces techniques volumétriques ont été utilisées chez la Vache, par Matthews, SwetT et Graves, I94I ; Baxter et al., I950 ; Andreae, I954; Hauptman et Svarcbeck, I956; Loppnow, I959; Jannerman, I960; Comberg et Schommlez, I96I ; Politiek, ig62 ; et chez la brebis par Ricordeau, MartiNET et DENAMUR, I 963 .

Iin dehors de ces appareils expérimentanx, de nombreux types de "Milk-Meter» sont actuellement construits en vue du contrôle des quantités totales de lait, soit chez l'exploitant, soit dans les laiteries. Il nous suffira de citer quelques principes de fonctionnement :

- compteur à basculement (Wilkowske, I956; Phiı.LIPS, I96r) ;

- pompe à diaphragme (MEAD et LABEN, I960) ;

- débitmètre à turbine ou à roue ovalisée, dont STEIn (I960) fait une description résumée.

Chez la brebis, le temps de traite étant légèrement supérieur à une minute et le volume de lait obtenu atteignant en moyenne un demi-litre, il nous a paru utile de concevoir un appareil qui tienne compte de ces particularités, c'est-à-dire suffisamment sensible et permettant l'enregistrement automatique des quantités de lait, selon un rythme nécessairement plus rapide que chez la vache. 
L'objet de cette note est :

- d'une part, de décrire deux appareils répondant à ces besoins,

- d'autre part, de présenter quelques résultats préliminaires n'ayant qu'une valeur indicative, car ils portent sur des laps de temps très courts et sur un nombre réduit d'animaux.

\section{II. - MATÉRIEL E'T TECHNIQUES}

\section{$\left.\mathrm{I}^{\circ}\right)$ Caractéristiques de la machine à traire}

- Marque : Alfa-Laval.

- Vide $: 33 \mathrm{~cm}$ de mercure.

- Vitesse de pulsation : $180 /$ minute.

- Rapport : $1 / \mathrm{r}$.

- Gobelet pesant 240 g environ sans canalisation.

- Corps du gobelet : I $44 \mathrm{~g}$; diamètre : $30 \mathrm{~mm}$; hauteur : 1 10 $\mathrm{mm}$; pipe inférieure : $63 \mathrm{~g}$.

- Caoutchouc : $33 \mathrm{~g}$; diamètre de l'entrée : $20 \mathrm{~mm}$; diamètre du corps : $20 \mathrm{~mm}$.

Les animaux sont traits sur un " carroussel " à $3^{2}$ places.

\section{$\left.2^{\circ}\right)$ Contrôle de la cinétique de la traite}

A.) Méthode volumétrique à l'aide d'un suiveur de niveau (SEFRAM).

Le lait de l'animal est recueilli dans un pot rigoureusement cylindrique muni d'une tubulure latérale en verre. Ia vitesse d'élévation du niveau du lait dans ce tube reflète donc très exactement la cinétique de remplissage du réservoir principal.

Le pot est placé le plus près possible de la mamelle, a fin de réduire l'erreur due à l'espace mort constitué par les canalisations.

Le suiveur de niveau (fig. I $a$ ) comprend :

a) Un chariot mobile composé d'une ampoule électrique fournissant un faisceau lumineux projeté sur une cellule photo-résistante. La tubulure latérale du pot trayeur est placée parallèlement aux rails du chariot. Elle se situe entre la lampe et la cellule photo-résistante. La variation de luminosité correspondant au passage du faisceau sur le niveau du lait agit sur la cellule. Le courant issu de celle-ci est amplifié, puis envoyé dans un servo-moteur qui corrige tout écart de position entre le chariot et le niveau du liquide.

b) Un curseur solidaire du chariot mobile se déplace le long d'un potentiomètre linéaire bobiné sur quartz. Si une tension est appliquée aux extrémités de ce potentiomètre on dispose entre le curseur et l'extrémité inférieure par exemple, d'une différence de potentiel dont les variations sont rigoureusement proportionnelles à la quantité de lait présente dans le pot.

Ces différences de potentiel sont enregistrées sur une table à déroulement MECI, à raison d'un pointage toutes les 4 secondes.

La précision de positionnement du curseur étant $\pm 0,2 \mathrm{~mm}$ et le diamètre du pot trayeur de I00 $\mathrm{mm}$; la précision de la mesure fournie par l'appareil est ainsi de $\pm \mathrm{I}, 35 \mathrm{ml}$.

\section{B.) Méthode pondérale à l'aide d'un dynamomètre électronique (SEXTA).}

Cet appareil (fig. $\mathrm{x} b$ ) est constitué par un anneau dynamométrique métallique sur lequel sont placées quatre jauges à fil résistant, associées en pont de Wheatstone. Cet anneau se déforme élastiquement sous l'influence des forces qui lui sont appliquées (le poids du pot trayeur et de son contenu-

Le pont, compensé de tous les effets parasites (température, flexion), constitue l'unité de mesure électrique sensible aux variations de poids du pot trayeur. 
On adjoint à ce traducteur de force un adapteur qui assure les fonctions suivantes : alimentation du pont de mesure par un courant stabilisé 5 volts continu ;

réglage du zéro électrique du traducteur (équilibrage initial du pont de mesure) ; réglage de la sensibilité ;

étalonnage (en grammes).

La tension de sortie proportionnelle au poids de lait présent dans le pot est appliquée à un enregistreur à déroulement du même type que celui précédemment décrit.

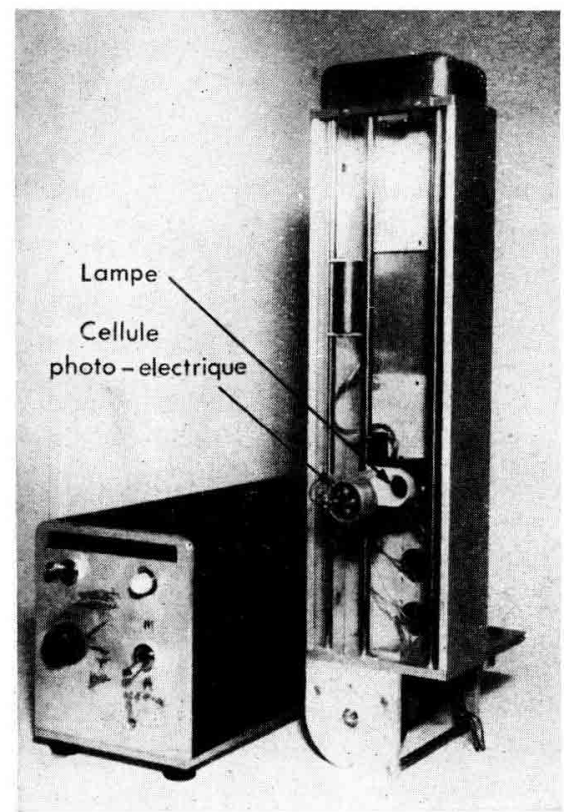

(a)

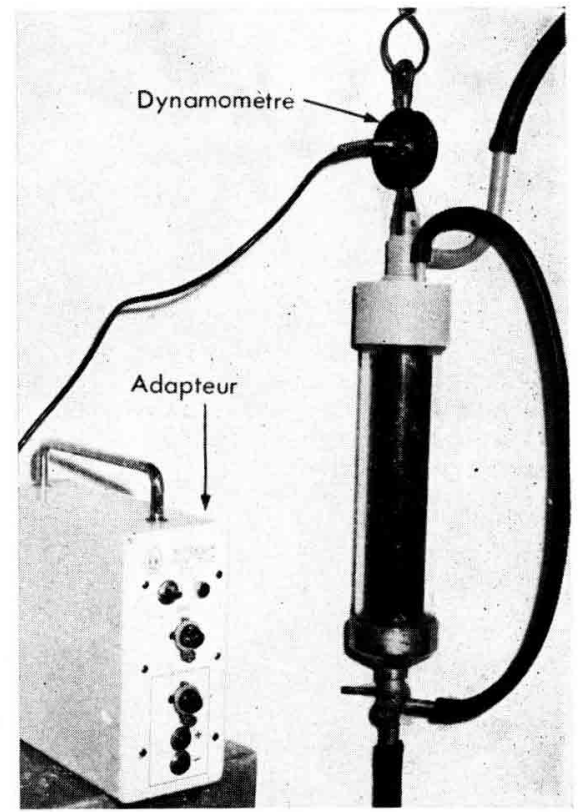

(b)

FIG. I - Les appareits de mesure

a) le suiveur de niveau

b) le dynamometre électronique

\section{$\left.3^{0}\right)$ Troupeau expérimental}

Nous avons contrôlé de la $4^{\mathrm{e}}$ à la $6^{\mathrm{e}}$ semaine de lactation 23 brebis (Préalpes du Sud, Frisonne et produits de croisements de ces deux races).

Ces brebis étant tirées au hasard dans le troupeau, leur âge (I à 8 ans), le numéro de leur lactation $\left({ }_{1}{ }^{\mathrm{re}} \dot{\mathrm{a}} 7^{\mathrm{e}}\right.$ ) et le nombre d'agneaux (I ou 2) sont aléatoires.

\section{$\left.4^{\circ}\right)$ Technique de traite et de contrôle}

Tous les animaux sont mis à la traite 24 à 48 heures après la mise bas. Les contrôles sont faits une fois par jour à la traite du soir. Les gobelets sont posés sans massage préalable; lorsque le lait cesse de couler dans le pot, le trayeur masse énergiquement la mamelle, les gobelets étant encore en place (égouttage machine), enfin, un trayeur spécialisé effectue un égouttage manuel.

\section{$\left.5^{\circ}\right)$ Définitions}

- Lait machine (en $\mathrm{ml}$ ) : volume du lait obtenu par la machine sans intervention du trayeur ;

-- égouttage machine (en $\mathrm{ml}$ ) : volume du lait obtenu à la machine lors du massage par le trayeur;

- égouttage manuel (en $\mathrm{ml}$ ) : volume du lait obtenu lors de la traite à la main après l'enlève. ment des gobelets ; 
- fin de la traite machine : elle a été fixée lorsque le débit devient inférieur à $5 \mathrm{ml} / 4 \mathrm{~s}$ avant l'égouttage machine;

— temps de traite machine (en secondes) : durée de la traite depuis la pose des gobelets jusqu’à la fin de la traite machine;

- débit moyen machine (en $\mathrm{ml} / 4$ secondes) :

C'est le rapport : $\frac{\text { lait machine en } \mathrm{ml} \times 4}{\text { temps de traite }}$

\section{III. - RÉSULTATS OB'TENUS AVEC LE SUIVEUR DE NIVEAU}

Io) ÉTUDE DE QUELQUES COURBES CARACTÉRISTIQUES

La figure 2 a donne un exemple des enregistrements obtenus. Avant le début de la traite, le pot est vide, les pointages successifs tracent une ligne verticale qui correspond au niveau zéro. L'arrivée du lait dans le récipient collecteur se traduit
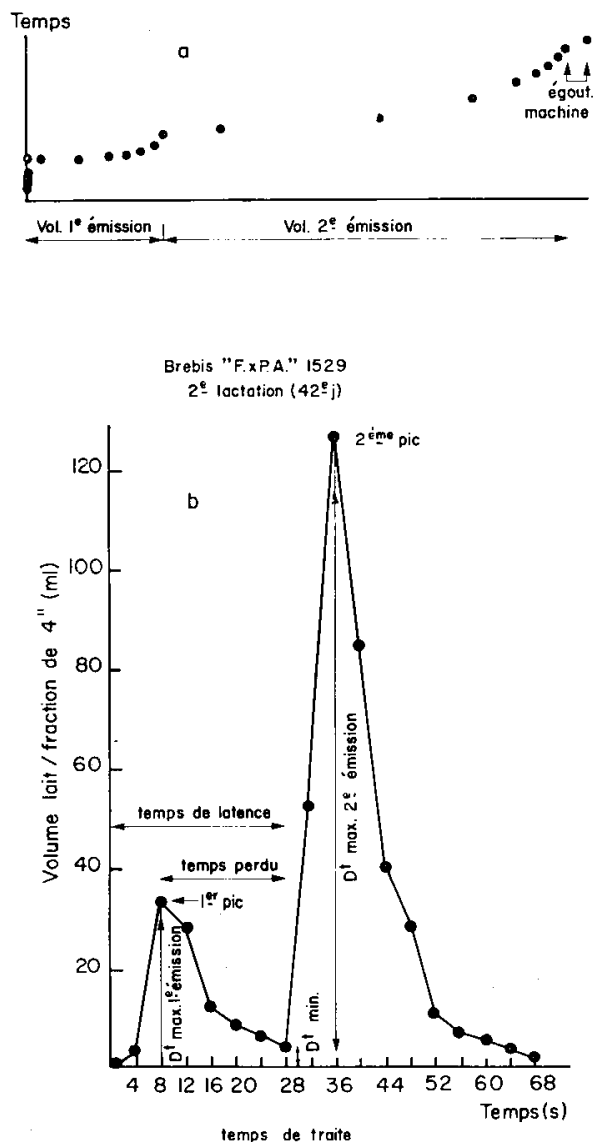

FIG. 2 - Enregistrement obtenu avec le suiveur de niveau

a) courbe du lait trait à l'instant $\mathrm{T}$

b) courbe exprimée en débit par fraction de 4 secondes 
par un déplacement vers la droite du système de pointage (courbe du lait trait à l'instant $\mathrm{T}$ ).

La même courbe peut être exprimée en débit par fraction de 4 secondes et prend l'aspect indiqué à la figure $2 b$, sur laquelle nous avons porté les définitions nouvelles imposées par nos résultats.
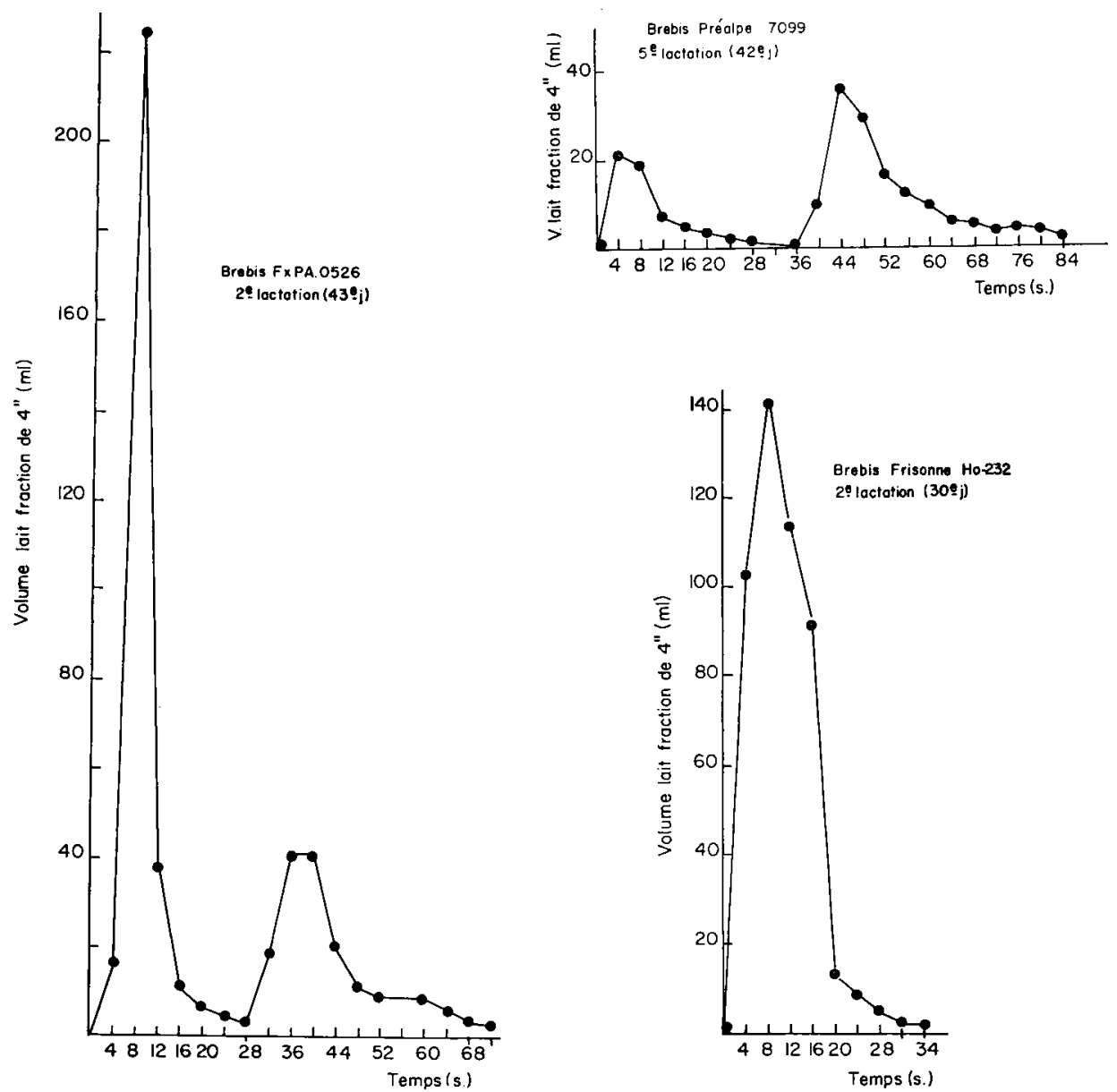

FIG. 3 - Exemple des variations individuelles : courbe de descente de lait de 3 brebis différentes

La figure 3 présente quelques exemples de courbes de descente que l'on peut classer en deux types suivant le nombre d'émissions de lait au cours de la traite.

$\mathrm{I}^{\mathrm{er}}$ cas: Courbes à 2 émissions de lait.

La première émission présente son débit maximum vers la $8^{\mathrm{e}}$ seconde et diminue graduellement pour, généralement, cesser vers la $24^{\mathrm{e}}$ seconde.

I a deuxième émission commence vers $1 \mathrm{a} 30^{\mathrm{e}}$ seconde, passe par un maximum 


\section{TABLEAU I}

Caractéristiques de traite des animaux à deux émissions de lait

\begin{tabular}{|c|c|c|c|c|}
\hline & \multicolumn{2}{|c|}{$\begin{array}{l}\text { Brebis Préalpes } \\
11 \text { animaux }\end{array}$} & \multicolumn{2}{|c|}{$\begin{array}{l}\text { Brebis croisées } \\
9 \text { animaux }\end{array}$} \\
\hline & Moyenne & Écart-type & Moyenne & Écart-type \\
\hline Lait total $(\mathrm{ml}) \ldots$ & 313 & 106,6 & 572 & 112,5 \\
\hline 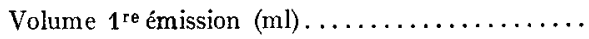 & 90 & 33,5 & 188 & 74,7 \\
\hline Volume $2^{\mathrm{e}}$ émission $(\mathrm{ml}) \ldots \ldots \ldots \ldots \ldots$ & 160 & 75,9 & 291 & 94,9 \\
\hline Lait machine $(\mathrm{ml}) \ldots \ldots \ldots \ldots \ldots \ldots \ldots \ldots$ & 251 & 91,94 & 480 & 104,7 \\
\hline Égouttage machine $(\mathrm{ml}) \ldots \ldots \ldots \ldots \ldots \ldots$ & 31 & 18,10 & 47 & 18,27 \\
\hline Égouttage manuel $(\mathrm{ml}) \ldots \ldots \ldots \ldots \ldots \ldots$ & 31 & 9,70 & 44 & 12,78 \\
\hline 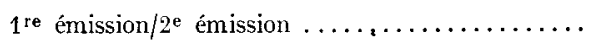 & 0,636 & 0,311 & 0,691 & 0,419 \\
\hline Lait $1^{\text {re }}$ émission $\%$ total $\ldots \ldots \ldots \ldots \ldots$ & 29,5 & 9,04 & 32,8 & 11,2 \\
\hline Lait $2^{e}$ émission $\%$ total $\ldots \ldots \ldots \ldots \ldots$ & 49,9 & 8,9 & 50,7 & 11,2 \\
\hline Égouttage machine $\%$ total .............. & 9,8 & 3,9 & 8,3 & 2,6 \\
\hline Égouttage manuel $\%$ total $\ldots \ldots \ldots \ldots \ldots \ldots$ & 10,3 & 3,1 & 7,8 & 2,1 \\
\hline Lait machine $\%$ total $\ldots \ldots \ldots \ldots \ldots \ldots$ & 79,6 & 6,01 & 83,6 & 4,25 \\
\hline Débit maximum $1^{\text {re }}$ émission $(\mathrm{ml} / 4 \mathrm{~s}) \ldots \ldots \ldots$ & 32,5 & 14,24 & 64,5 & 29,81 \\
\hline Débit minimum $(\mathrm{ml} / 4 \mathrm{~s}) \ldots \ldots \ldots \ldots \ldots$ & 1,9 & 1,58 & 5,8 & 3,75 \\
\hline Débit maximum $2^{\mathrm{e}}$ émission $(\mathrm{ml} / 4 \mathrm{~s}) \ldots \ldots \ldots$ & 34,0 & 14,9 & 70,8 & 26,5 \\
\hline 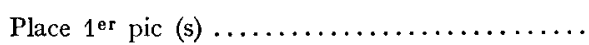 & 7,3 & 1,44 & 9,4 & 2,47 \\
\hline Temps de latence $(s) \ldots \ldots \ldots \ldots \ldots \ldots \ldots$ & 29,8 & 2,75 & 29,0 & 5,24 \\
\hline 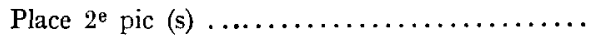 & 39,4 & 2,59 & 41,0 & 4,63 \\
\hline Temps de traite $(s) \ldots \ldots \ldots \ldots \ldots \ldots \ldots$ & 61,5 & 13,39 & 64,0 & 5,47 \\
\hline Temps perdu $(s) \ldots \ldots \ldots \ldots \ldots \ldots \ldots \ldots$ & 22,6 & 3,33 & 19,5 & 4,56 \\
\hline Débit moyen $(\mathrm{ml} / 4 \mathrm{~s}) \ldots$ & 16,39 & 5,58 & 30,22 & 7,52 \\
\hline
\end{tabular}


aux environs de la $40^{\mathrm{e}}$ seconde, puis s'estompe peu à peu, au-delà d'une minute après la pose des gobelets.

Le tableau I résume les caractéristiques de traite des 20 animaux contrôlés et qui appartiennent à ce groupe.

De l'ensemble de ces résultats, il ressort que :

a) il existe de grandes différences individuelles dans la répartition du lait entre les différentes fractions. Il en est de même pour les débits ; toutefois, à des productions importantes correspondent des débits importants. Par contre, comme l'indique la figure 4 et les coefficients de répétabilité du tableat 2 , chaque animal semble réagir d'une manière stable au cours des traites successives faites dans des conditions standard.

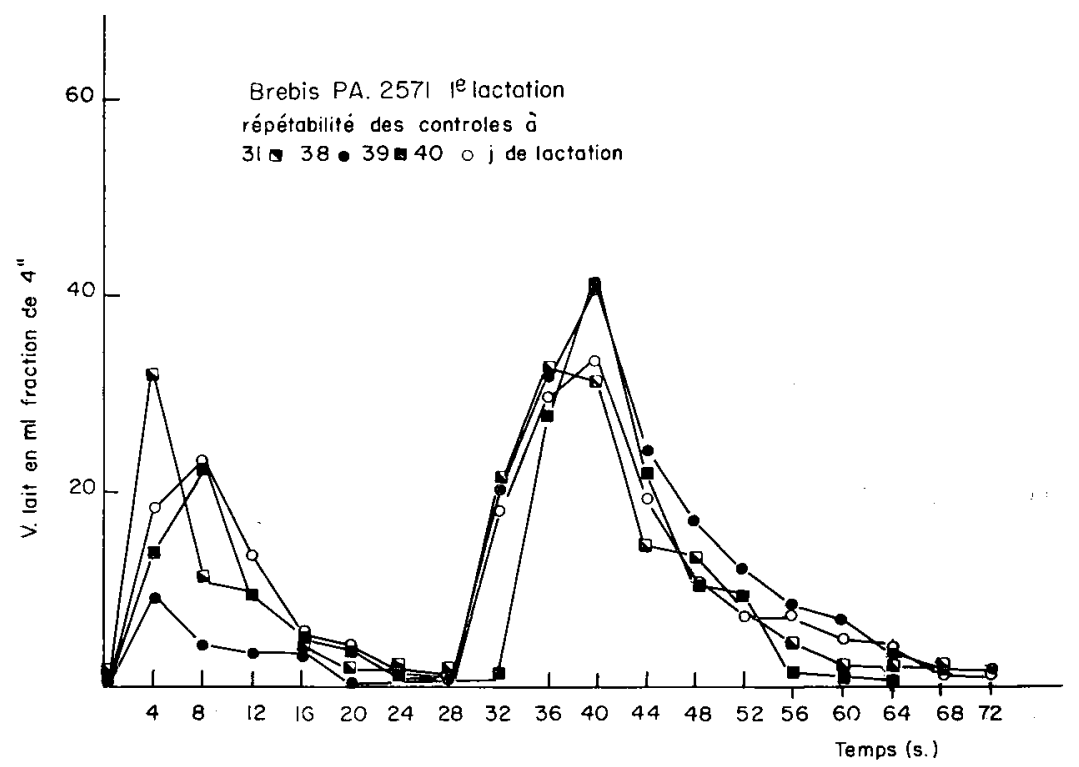

Fig. 4 - Répélabilité des courbes chez un même animal.

b) La place des différents phénomènes dans le temps paraît être indépendante des qualités laitières de l'animal.

c) Le temps perdu entre le $\mathrm{I}^{\mathrm{er}}$ pic et l'apparition de la seconde émission corres: pond environ à $\mathrm{I} / 3$ du temps de traite, le débit entre les deux pics devenant alors très faible ( $\mathrm{I}, 9 \mathrm{ml} / 4 \mathrm{sec}$. en moyenne).

d) Il faut donc attendre environ 30 secondes entre la pose des gobelets et l'apparition de la $2^{\mathrm{e}}$ émission. Cette valeur est de l'ordre de grandeur des temps de latence signalés chez la brebis pour le réflexe neuro-endocrinien d'éjection (WhitTLEstonE; I957).

$2^{\mathrm{e}}$ cas : Courbe à une émission de lait.

Une brebis Frisonne et deux brebis issues de croisement Frisonne $\times$ Préalpes présentent une courbe de ce type (fig. 3). 


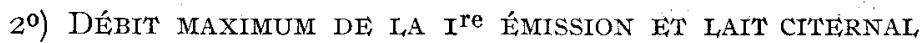

Chez les brebis présentant deux émissions, la relation entre le lait présent dans la citerne et le débit maximum du I ${ }^{\mathrm{er}}$ pic est probablement indépendante de l'intensité du réflexe neuro-endocrinien d'éjection. Elle ne dépendrait donc que de la morphologie et de la motricité de la citerne et du trayon.

TABLEAU 2

Coefficients de répétabilité

\begin{tabular}{|c|c|c|c|}
\hline & $\begin{array}{l}\text { Brebis } \\
\text { Préalpes }\end{array}$ & $\begin{array}{l}\text { Brebis } \\
\text { croisées }\end{array}$ & $\begin{array}{l}\text { Ensemble des } \\
\text { animaux }\end{array}$ \\
\hline Volume $1^{\text {re émission }}$ & & & \\
\hline$\overline{\text { Volume } 2^{\text {e }} \text { emission }} \cdots \cdots \cdots \cdots \cdots \cdots \cdots \cdots \cdots \cdots$ & 0,753 & 0,975 & 0,873 \\
\hline Volume $1^{\text {re }}$ émission $(\%$ du volume total $) .. \ldots \ldots \ldots$ & 0,862 & 0,962 & 0,916 \\
\hline Volume $2^{\mathbf{e}}$ émission $(\%$ du volume total) $\ldots \ldots \ldots \ldots$ & 0,664 & 0,957 & 0,805 \\
\hline Temps perdu $\ldots \ldots \ldots \ldots \ldots \ldots \ldots \ldots \ldots \ldots \ldots$ & $0,368(\mathbf{1})$ & $0,364(\mathbf{1})$ & $0,453(1)$ \\
\hline Temps de traite $\ldots \ldots \ldots \ldots \ldots \ldots \ldots \ldots \ldots \ldots \ldots$ & 0,892 & 0,657 & 0,822 \\
\hline Temps de latence $\ldots \ldots \ldots \ldots \ldots \ldots \ldots \ldots \ldots \ldots \ldots \ldots \ldots$ & 0,860 & 0,536 & 0,669 \\
\hline 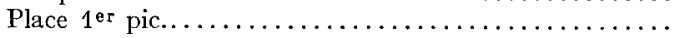 & $0,144(1)$ & $0,298(\mathbf{l})$ & $0,348(1)$ \\
\hline 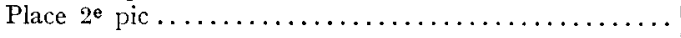 & 0,691 & 0,992 & 0,753 \\
\hline 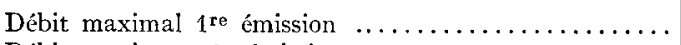 & 0,592 & 0,364 & 0,557 \\
\hline Débit maximum $2^{\mathrm{e}}$ émission $\ldots \ldots \ldots \ldots \ldots \ldots \ldots$ & 0,750 & 0,091 & 0,887 \\
\hline 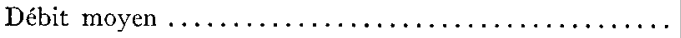 & 0,895 & 0,448 & 0,875 \\
\hline
\end{tabular}

(1) Pour les valeurs relativement faibles de ces coefficients on doit tenir compte du fait qu'il n'est possible d'apprécier le début de la traite qu'à \pm 4 secondes, ce qui est une erreur importante en p. 100 de la valeur absolue.

Notre étude portant sur 20 animaux, la relation que nous constatons peut se décomposer en deux phases (fig. 5).

\section{- Pendant la première:}

la quantité de lait citernal restant faible, la liaison entre volume et débit est linéaire et du type $y=0,482 x-4,25(y=$ débit maximum, $x=$ lait citernal $)$.

\section{- Pendant la seconde:}

Le débit croît beaucoup plus lentement lorsque le volume citernal augmente. Selon la limite inférieure que l'on fixe à ce volume pour cette seconde phase, la relation peut être :

- soit linéaire avec un coefficient de régression plus faible que précédemment $(y=0,205 x+40,2)$,

- soit exponentielle. 
Quelle qu'en soit 1'expression mathématique, ces résultats plaident en faveur du rôle limitant joué par l'élasticité de l'orifice du trayon dans la mise en évidence des débits maximum.

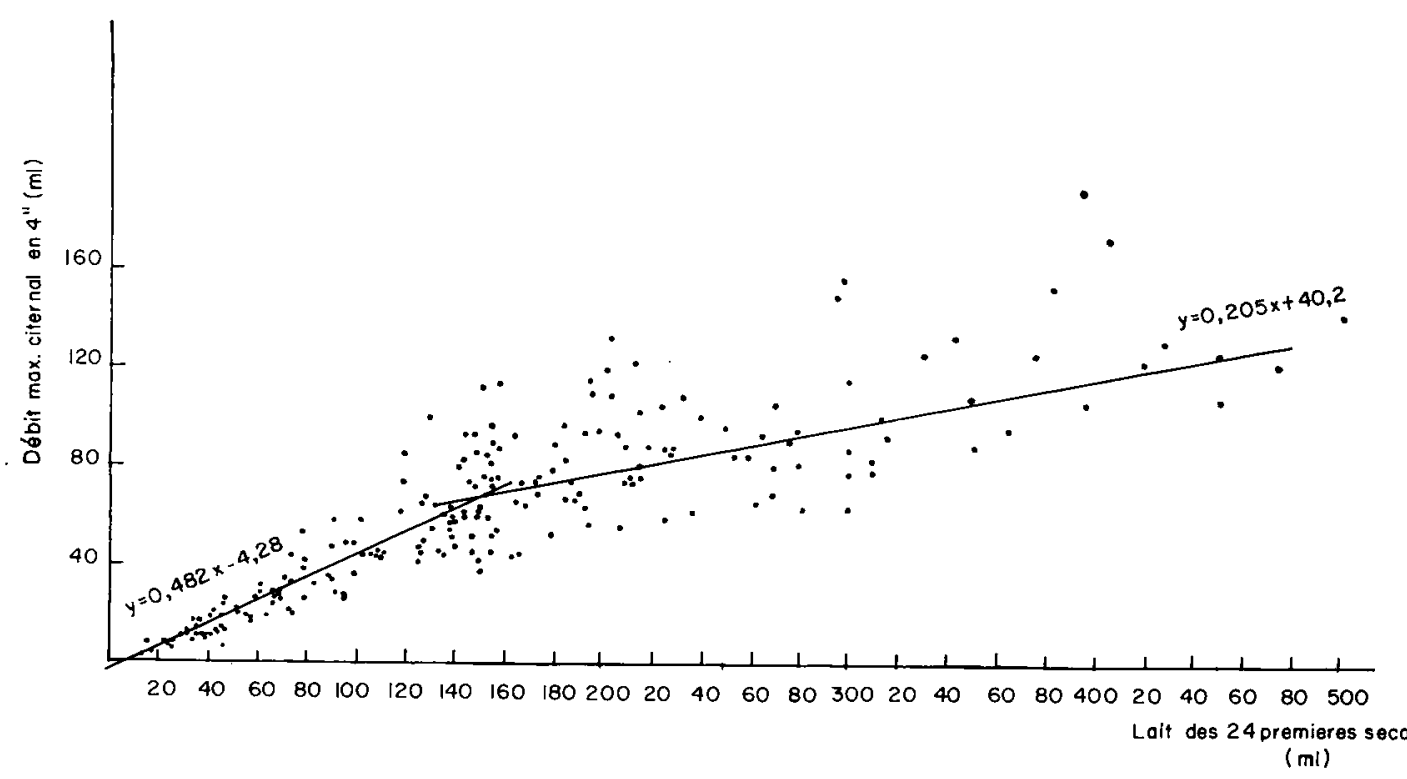

FiG. 5. - Relation entre le débit maximum de la première émission et le volume de lait citernal

\section{IV. - RÉSULTATS OBTENUS AVEC LE TRADUCTEUR DE FORCE}

Les courbes de descente de lait obtenues avec le traducteur de force sont tout à fait semblables aux enregistrements précédents.

Néanmoins, le vide pulsé de la machine à traire provoquant de petites trépidations parasites, nous pensons qu'à des fins expérimentales, il est préférable d'utiliser le suiveur de niveau.

\section{V. - DISCUSSION ET CONCLUSION}

Io) Les appareils de mesure.

Les deux appareils que nous venons de décrire nous donnent entière satisfaction, quoique nous portions notre préférence sur le suiveur de niveau pour les raisons que nous venons de donner. Il y a tout lieu de croire qu'un tel système pourrait être utilisé chez la vache dans des buts expérimentaux semblables. 
Dans l'avenir, pour permettre un dépouillement rapide, la chaîne de mesure est à concevoir ainsi :

- an ou plusieurs capteurs;

- un commutatetır de voies permettant d'aiguiller les mesures à effectuer sur les différents capteurs (I cycle toutes les $4 \mathrm{sec}$.).

- un voltmètre digital ;

- une machine imprimante ou un ensemble transcodeur-perforatrice de bandes.

\section{$\left.2^{\circ}\right)$ Les premiers résultats.}

Les deux pics d'émission que nous constatons précisent les premiers résultats obtenus par Bosc (I962), Ricordeau, Martinet, Denamur (I963). Compte tenu des temps de latence signalés précédemment ces deux pics peuvent être interprétés comme :

- une phase d'écoulement du lait citernal qui durerait moins de 24 secondes,

- une phase d'écoulement du lait alvéolaire chassé entre la $30^{\mathrm{e}}$ et la $60^{\mathrm{e}}$ seconde par le réflexe neuroendocrinien d'éjection.

Ces deux émissions de lait sont séparées par un laps de temps pendant lequel la machine à traire travaille à vide. Cette " traite à blanc " est à étudier car elle peut constituer un danger du même ordre que celui encouru lorsqu'en fin de traite on laisse les gobelets trop longtemps sur des quartiers vides.

Il sera également intéressant de vérifier individuellement, d'une part, tout au cours de la lactation, d'autre part, en faisant varier artificiellement le volume de lait citernal, si le sphincter limite ou non le débit-lors de la traite (BAXTER et al.,

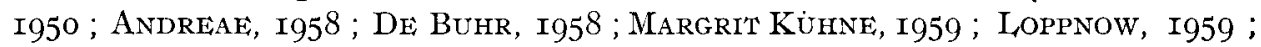
Johansson et Malven, I960; Barczinski, I96o, etc.).

L'un des principaux problèmes soulevés par ces premiers résultats est de savoir pour quelles raisons certains animaux sont capables de donner très rapidement tout leur lait en une seule émission. Ce type de courbe est-il à rapprocher de celles obtenues avec une méthode moins précise par GRINFELD, VolCANI et Eyal chez la Brebis Awassi? Il est probable que dans notre cas, le lait contenu dans les alvéoles s'est déversé dans la citerne avant le début de la traite. Parmi les nombreuses hypothèses que l'on peut formuler concernant la cause de ce transfert, nous donnerons les deux suivantes :

- chez certains animaux possédant de larges canaux galactophores et une grande citerne (BARCZINSKI, I960), le lait ne serait pas retenu au niveau des alvéoles et s'écoulerait au fur et à mesure de sa sécrétion. Ce phénomène pourrait être renforcé par le frottement des pattes arrière sur la glande entretenant ainsi un massage continuel entre les traites;

- les signaux acoustiques et visuels lors de l'arrivée dans la salle de traite (KOKORINA, I955; HoLTHOFF, I955) joueraient le rôle de stimulus conditionnel pour le réflexe neuro-endocrinien d'éjection du lait.

Il est utile de souligner l'intérêt zootechnique que présente la recherche de souches capables de fournir naturellement ou par une technique de traite appropriée une seule émission de lait. Le gain de temps obtenu pouvant être de l'ordre 
de 20 secondes par traite et par bête, ceci représente, comme le mentionnent GrivFELD, VOLCANr et EYAL (I959), une économie de 75 journées de travail par campagne laitière sur un troupeau de 300 brebis.

$$
\text { Rę̧u pour publication en mars } 1964 .
$$

\section{REMERCIEMENTS}

Nous tenons à remercier la société SEFRAM (Paris) la société Alfa-Laval et la société MECI (Paris), ainsi que la société SEXTA (Bagneux) pour l'aide technique qu'ils nous ont apportée dans la réalisation de ce travail.

\section{SUMMARY}

\section{DESCRIPTION OF TWO TYPES OF APPARATUS FOR AUTOMATIC RECORDING MILK FLOW DURING MACIINE MILKING. FIRST RESULTS WITH EWES}

Milk flow was recorded automatically during machine malking of 23 ewes of different breeds in about the first month for lactation.

Two methods were used :

I. A volumetric method with a level follower (fig. $\mathrm{I} a$ ).

This apparatus consists of a mobile carriage carrying a lamp, a photoelectric cell and a moving indicator.

The beam from the lamp is directed to the photoclectric cell and is interrupted by the milk in the glass lateral tube of the jar of the milking machine. Difference in position between the carriage and the level of milk is corrected by a servo-motor.

The indicator moves along a linear potentioneter fed with direct current. Every 4 seconds the difference in potential between the lower and middle points of the potentiometer is recorded on a moving sheet.

\section{A gravimetric method with an electric dynamometer (fig. I $b$ ).}

This apparatus consists of a dynamometric ring on which are placed 4 gauges with resistance wires attached to a Wheatstone bridge. The change in shape of the ring under the weight of the jar of the milking machine and its contents is measured : the electrical output, proportional to the weight of milk, is recorded as above.

The results were as follows :

Three ewes gave all their milk in a single phase of let down. Thus milking was completed in 30 seconds (fig. III).

In contrast, there were two distinct phases in 20 ewes (fig. III). In thoses case the first phase would correspond to the flow of cisternal milk. This flow was greatest about the 8th second after the teat-cups had been put in place, and then gradually diminished and stopped after about $24 \mathrm{se-}$ conds.

The second phase would be the flow of alveolar milk caused by neuro-endocrine reflex. It began after about 20 seconds and lasted for a little longer than the first minute of milking.

There were wide differences between individuals in the distribution of milk between the different fractions (table. I), but the shape of the curve for any one animal remained the same from day to day (table. II, fig. IV).

The elasticity of the teat canal would affect the flow of milk. 


\section{RÉFÉRENCES BIBLIOGRAPHIQUES}

Ace D. L., Thoel II. W., Kesler E. M., Clominger W, H., i959. Device for measuring rate of milk flow. J.Dairy Sci., 42, 876-877.

ANDREAE U., 1954. Ein Weg zur objektiven Ermittlung der Melkbarkeit von Kühen. Zïchtungskunde, 26, $\mathrm{x} 43^{-1} 46$.

ANDreat U., 1958. Messungen am Zitzenkanal von Kühen zur Ermittlung der Melkbarkeit. Z. Tierz. Züchlbiol., 71, 289-298.

BARCzynsky G., I960. Ein experimenteller Beitrag zu den anatomisch-funktionellen Grundlagen der Melkeigenschaften des Rindes. Inaug. Diss. Tierärztl. Hochsch. Hannover, I-48.

Baxter E. S., Clarke P. M., Dodi F. H., Foot A. S., i950. Factor affecting the rate of machine milking. J. Dairy Res., 17, I I $7^{-127 .}$

Beck G. H., Fryer H. C., Roark D. B., I95I. Use and interpretation of milk flow curves in measuring variations in the response of cows to machine milking. J. Dairy Sci., 34, 58-67.

Beigel M., I955. Der Einfluss der Bullen auf die Schnelligkeit der Milchhergabe seiner Nachkommenschaft. Inaug. Diss. Doct. Med. Vet. Hannover.

BLAU, von Günther, I956. Beiträge zum Studium des Milchentzuges beim Rind. Züchtungskunde, 28, 285 298.

Bosc J., ig6z. Adaptation du système "Arête de Poisson "à la traite mécanique des brebis. Cah. Ing. Agron., 164, 29-36.

Buhr W. de, I958. Der Einfluss einer Anaesthesie des Strichkanals und der Zisterne auf die Melkgeschwindigkeit und auf den Milchdruck. Inaug. Diss. Tierärzil. Hochsch. Hannover, 1, 40.

Caruolo F. V. Dembiczak C. M., Mochrie R. D., Johnson R. E., Eaton H. D., Spielman A. A., i 955. Effect of vacuum level and milking duration on Guernseys and Holsteins differing with respect to lactation number and status of udder health. II. Milk yield, milking time, and rate of milk flow. J. Dairy Sci., 38, I $283-1292$.

Clough P. A., Dodd F. H., r957. The relationships between milk secretion and the rate of milking by machine. J. Dairy Sci., 24, I $52-156$.

Comberg, G., von Zschommler H. G., ig6r. Das mittlere und höchste Minutengemelk als Ausdrucksformen der Melbarkeit von Kuheutern. Züchlungskunde, 33, I3-3I.

Dodd F. H. Foot A. S., Henriques E., Neave F. K., i95o. Experiments on milking technique. J. Dairy Res., 17, I I 7-1 27.

Donald H. P., I960. Genetical aspects of maximum rate of flow in Holstein-Friesian dairy cattle. J. Dairy Res., 27, 361-37.

Foot A. S., 1935. The rate of milking by machine. J. Dairy Res., 6, 3근ㄷ․

GaInes W. L., 1927. Differences measured in the rate at which cows milk. Ill. Agr. Expt. Sta. 4 th Ann. Rept., 158.

Grégoire A. T. Mochrie R. D., Elliot F. I., Eaton H. D., Spieldman A. A., i954- Effect of vacuum level and milking duration on milk production, milking time and rate of milk flow in mastitis free first calf heifers. J. Dairy Sci., 3\%, 276-283.

Grinfeld J., Volcani R., Eyal A., i959. Quelques observations sur l'adaptation de la brebis Awassi à la traite mécanique (en hébreu). Hanoked, 42 .

Hauptman J., Svarcber J., I956. Nouveau système pour la détermination de la vitesse de traite (en tchèque). Za Socialist Zemed, 6, гоб--1о7.

Holtiof F., I956. Untersuchungen über die Schnelligkeit der Milchhergabe beim Rind. Inaug. Diss. Hannover.

Hupfauer M., 1956. Der Einfluss der Druckwechselzeiten von Pulsatoren auf die Melkleistung. Landtechnik. München, 6, 26-31, in Dairy Sci. Abstr., 18, 819-820.

Hupp E. W., Plum M., 1957. Normal variations in the maximum rate of milk flow in Holstein-Friesian dairy cattle. J. Dairy Sci., 40, 624.

JANNERMANN I., Ig60. Beinflussung von Eutereigenschaften durch die Vatertiere. Tierzucht, 14, I02-I07.

Johansson I., Malven P., I960. The influence of yield, udder pressure, size of teats and of the teat orifice on the rate of milking. Z. Tierz. Ziichtbiol., 74, I-13.

Kokorina E. P., 1955. La nature du réflexe d'éjection du lait chez les vaches avec différentes particularités typologiques de l'activité nerveuse supérieure (russe). Dokl. Akad. Nauk. SSSR., 108, 746-749.

Kunne M., I959. Strukturverhältnisse im Bereich des Zitzenstrichkanals und ihre Beziehungen zur Melkbarkeit. Inaug. Diss. Tieräratl. Hochsch. Hannover, i à zo.

LOPPNow, von H., I959. Über die Abhängigkeit der Melkbarkeit vom Bau der Zitze. Disch. Tierärztl. Wschr., 66, $88-97$. 
Matthews C. A., Shaw J. M., Weaver E., 1928. The economy and efficiency of a milking machine. Iowa Agr. Expt. Sta. Bull., 248.

Matthews C. A., Swetr W. W., Graves R. R., 1941. Milk yields and milking rates of the individual quarters of the dairy cow udder. U.S.D. A. Technical Bull., 827, I-32.

Mead S. W., LABeN R. C., I960. Accuracy determination of a new milk metering and sampling device. J. Dairy Sci., 43, 895 .

Murray A. J. W., I960. An apparatus for recording milk flow curves of cows milked by machines. $Q d$. J. Agric. Sci., 17, 147-1 53, in Dairy Sci. Abstr., 23, 474.

Phillirs D. S. M., I96i. A milk meter for New Zealand. N. Z. Dairy Exper., 37, 65-66, in Dairy Sci. Abstr., 24, 22.

Politiek R. D., 1962. Observations sur l'analyse de la facilité de traite sur la variabilité chez les vaches ainsi que sur l'héritabilité de ce caractère. Bull. T'ech. Ing. Serv. Agric., 166, 9-ı6.

Ricordeau G., Martinet J., Denamur R., ig63. Traite à la machine des brebis Préalpes du Sud, importance des différentes opérations de la traite. Ann. Zootech., 12, 203-225.

SANDVIK O., I957. The relationships between milking rate and milk yield. J. Dairy Res., 24, 316-320.

Schwenzer H., 1958. Abhängigkeit der Melkgeschwindigkeit von der Euterausbildung des Höhenfleckviehs. Inaug. Diss. Doct. Med. Vet. Hannover.

Stein F., 1960. Liquid meters for fluid milk measurement. Amer. Milk. Rev., 22, 32-II 2.

Stewart W. E., Schultz L. H., i956. Effect of vacuum and pulsation rate on rate of milking. J. Dairy Sci., 39, 926-927.

Sych E., 1961. Zur Bestimmung der Melkgeschwindigkeit. Tierzucht, 15, 103-105.

Viljoen G. D., Swart J. C., I961. An improved method for determining rate of milk flow. S. Afric. J. Agric. Sci., 4, 639-642.

Whittlestone W. G., Phillips D. S. M., I953. Automatic apparatus for drawing the milk ejection curves of dairy cows under controlled milking conditions. J. Dairy Res., 20, 319-326.

WhitTlestone W. G., 1957. Intramammary pressure in the lactating ewe. I. The nature of the pressure change under standard conditions. J. Dairy Res., 24, I65-г 70.

Whittlestone W. G., ig6o A simple remote controlled milk flow recorder. Aust.J.Dairy Techn., 15, I97I99.

WrLkowske H. H., 1956. A new milk metering device. Hoard's Dairym., 101, $77^{2-773 . ~}$ 